\title{
Genetic variability of high molecular weight glutenin subunits in bread wheat from continental Portugal, Madeira and Canary Islands
}

\author{
Teresa M. M. dos Santos • Humberto Nóbrega • José Filipe Teixeira Ganança • \\ Emanuel Silva • Desire Afonso - Abílio F. M. Gutiérres - Jan J. Slaski • \\ Manhaz Khadem • Miguel Â. A. Pinheiro de Carvalho
}

Received: 28 April 2011/ Accepted: 23 September 2011/Published online: 12 October 2011

(C) Springer Science+Business Media B.V. 2011

\begin{abstract}
The genetic variability of high molecular weight glutenin subunits (HMWGS) composition at the Glu-l loci in bread wheat (Triticum aestivum L.) was studied electrophoretically using the SDS-PAGE in 3,470 individuals representing 159 populations originated from the Canary Islands (Spain), the Archipelago of Madeira (Portugal) and the continental Portugal. A total of 25 alleles were detected, resulting in 69 different allele combinations. The geographical distribution of the high molecular weight glutenin alleles confirms historical data regarding circulation of
\end{abstract}

T. M. M. dos Santos · H. Nóbrega ·

J. F. T. Ganança · E. Silva · J. J. Slaski ·

M. Khadem - M. Â. A. Pinheiro de Carvalho

ISOPlexis Germplasm Bank, University of Madeira, 9000-390 Funchal, Portugal

J. F. T. Ganança $(\bowtie)$

ISOPlexis Germplasm Bank, University of Madeira, 9000-081 Funchal, Portugal

e-mail: jofitei@gmail.com

D. Afonso

Centro de Conservación de la Biodiversidad Agrícola de

Tenerife, Tacaronte, Tenerife, Spain

A. F. M. Gutiérres

Servicio de Agricultura Ganaderia y Pesca, Cabildo de La Palma, Avenida de Europa no. 9, Buenavista, 38710

Breña Alta, Spain

\section{J. J. Slaski}

Bioresource Technologies, Alberta Innovates Technology Futures, Vegreville, AB T9C 1T4, Canada wheat germplasm between the Iberian Peninsula and Madeira and between Madeira and the Canary Islands and vice versa.

Keywords Canary Islands · Genetic variability · High molecular weight glutenin subunits · Madeira Island · Portugal

\section{Introduction}

Wheat was introduced to the Atlantic Archipelagos of Madeira and Canary five hundred years ago. Madeira was colonised first in the beginning of the fifteenth century. The Portuguese settlers brought wheat that was cultivated on the island to provide food for steadily growing local population. During the first six decades Madeira farmers produced high volumes of the crop and the surplus of wheat grain was exported to the Azores, Canary and Maghreb and thus was an important resource for the Portuguese explorations along the West Coast of Africa (Vieira 1988). From the seventies of the fifteenth century, a great portion of the arable land of Madeira was converted to production of new lucrative crops including sugar cane and grapes, while wheat plots were relegated to the less fertile land. As a consequence, wheat yields drastically declined and crop production was insufficient to feed the Madeira population. This situation imposed an 
urgent need to increase area of land devoted to wheat cultivation. Therefore, new production areas were developed on the neighbouring Archipelagos of Canary and Azores (Vieira 1984; Freitas de Meneses 1995). The commercial flow of wheat seeds among the Azores, Canary and Madeira Archipelagos over the course of several centuries resulted in the accumulation of high wheat diversity on these islands (dos Santos et al. 2009).

Seed storage proteins are a result of genome expression and contain extensive genetic variation in wheat landraces (Chaparzadeh et al. 2008; Terasawa et al. 2009). Therefore, they are considered to be good criteria for genetic diversity studies (Porceddu et al. 1998). The high molecular weight glutenin subunits (HMWGS) are storage proteins synthesized in the seeds of wheat and related species (Lawrence and Shepherd 1981; Shewry et al. 1995). The HMWGS are encoded by the Glu-l loci located on the long arms of the homoelogous group one chromosomes, with each locus comprising two linked genes encoding $\mathrm{x}$ and y-types subunits (Lawrence and Shepherd 1981, Payne 1987, Wan et al. 2005). Consequently, three loci (Glu-Al, GluBl and Glu-Dl) encoding six HMWGS are present in hexaploid wheat (Triticum aestivum, $2 \mathrm{n}=6 \mathrm{x}=42$, AABBDD), which originated 10,000 years ago from natural hybridization between tetraploid wheat $(2 \mathrm{n}=4 \mathrm{x}=28$, AABB $)$ and diploid Ae. tauschii $(2 \mathrm{n}=2 \mathrm{x}=14$, DD) (Feldman et al. 1995; Feldman 2000). The subunits differ in the molecular weight (higher for $\mathrm{x}$-type), number of cysteine residues, and repetitive motifs. Silencing of specific genes results in variation in the number of expressed subunits from three to five, while allelic variation in subunits encoded by the expressed genes leads to polymorphism of x-type and y-type subunits, which can be separated by the SDS-PAGE (Payne and Lawrence 1983; Payne et al. 1987; Vallega 1988; Feng et al. 2004). The HMWGS have been successfully used to assess the genetic diversity of wheat germplasm from different countries (Lawrence 1986; Nevo and Payne 1987; Lagudah et al. 1987; Morgunov et al. 1990; Caballero et al. 2001; Branlard et al. 2003; Hua et al. 2005; Tohver 2007; Shan et al. 2007; Li et al. 2009).

The main goal of the present study was to analyze the HMWGS composition of a collection of wheat accessions collected from the continental Portugal, Madeira and the Canary Islands.

\section{Materials and methods}

Plant material

A total of 159 Triticum aestivum L. accessions were used in this study (Table 1), among them 22 accessions originated from the continental Portugal (the Vasconcellos (1933) collection), 63 from Madeira and 74 from the Canary Islands. The seeds were provided by the ISOPlexis Gene Bank at the University of Madeira, Centro de Biodiversidad de Tenerife, Banco de Germoplasma de España, Cabildo de La Palma and Maria Teresa Carvalho e Vasconcelos, Instituto Superior de Agronomia (ISA). The standard wheat cultivars (Gabo, Marquis, Payne, Atlas 66, Federation, Florida 301, Cheyenne, Florence, David, Forlani, Champlein, Atlas 50, Chinese Spring) were kindly provided by Dr. Harold Bockelaman from the National Small Grains Collection, USDA-ARS.

\section{SDS-PAGE analysis}

Proteins were extracted from individually crushed endosperm seeds wholemeal. Samples $(20 \mathrm{mg})$ were extracted once with $1 \mathrm{ml}$ dimethyl sulfoxide (DMSO) and twice with $1 \mathrm{ml} 50 \%$ propan-1-ol to remove gliadin, albumin and globulin proteins (mixing each time on a vortex mixer for $10 \mathrm{~s}$ and centrifuging $10 \mathrm{~min}$ at $16,000 \mathrm{~g}$ ). The full range of glutenin subunits was then extracted at $65^{\circ} \mathrm{C}$ for 30 min with $200 \mathrm{ml}$ of $1 \%$ SDS solution containing $1 \%$ dithiothreitol (DTT) followed by centrifugation for $10 \mathrm{~min}$ (Uthayakumaran et al. 2006).

Proteins were fractionated by electrophoresis in a vertical SDS-PAGE gel in a discontinuous Tris-HClSDS buffer system (pH 6.8/8.8) following the ISTA Standard Method for the SDS-PAGE (Cooke 1992). The gels were stained with Coomassie blue and visually analysed for allele identification and compared with the patterns of known genotypes (standards). The nomenclature of the HMWGS was followed as proposed by Payne and Lawrence (1983).

Data analysis

The following genetic variability parameters were calculated for all the populations using the POPGENE 1.32 program (Yeh and Boyle 1997): observed number of alleles per locus (na), effective number of alleles 
Table 1 Plant material and allelic composition of GLU-1 alleles

\begin{tabular}{|c|c|c|c|}
\hline Accession number & Common name & Origin & Alleles \\
\hline 2943 & Egípcio & Mainland (Portugal) & $\mathrm{b} ; \mathrm{d} ; \mathrm{g}$ \\
\hline 3100 & Tremês branco & Mainland (Portugal) & $\mathrm{b} ; \mathrm{d} ; \mathrm{a}$ \\
\hline 3085 & Rieti & Mainland (Portugal) & $\mathrm{a} ; \mathrm{b} ; \mathrm{d}$ \\
\hline 3526 & Mocho de esp. Branca & Mainland (Portugal) & $\mathrm{b} ; \mathrm{f} ; \mathrm{g}$ \\
\hline 17712 & Serrano & Mainland (Portugal) & $\mathrm{a} ; \mathrm{d} ; \mathrm{a}$ \\
\hline 2936 & Mestiço & Mainland (Portugal) & $\mathrm{a} / \mathrm{b} ; \mathrm{e} / \mathrm{g} ; \mathrm{a}$ \\
\hline 3643 & Precoce & Mainland (Portugal) & $\mathrm{b} ; \mathrm{d} / \mathrm{f} / \mathrm{g} ; \mathrm{a} / \mathrm{d}$ \\
\hline 2984 & Funchal & Mainland (Portugal) & $\mathrm{b} ; \mathrm{a} ; \mathrm{a} / \mathrm{h}$ \\
\hline 7940 & Ribeiro & Mainland (Portugal) & $\mathrm{b} ; \mathrm{d} ; \mathrm{g}$ \\
\hline 7938 & Almadense & Mainland (Portugal) & $\mathrm{a} ; \mathrm{an} ; \mathrm{a}$ \\
\hline 7501 & Saloio & Mainland (Portugal) & $\mathrm{a} / \mathrm{b} ; \mathrm{an} / \mathrm{g} ; \mathrm{a}$ \\
\hline 3136 & Transmontano & Mainland (Portugal) & $\mathrm{b} ; \mathrm{b} ; \mathrm{a}$ \\
\hline 3172 & Ideal & Mainland (Portugal) & $\mathrm{b} ; \mathrm{a} / \mathrm{ag} ; \mathrm{g}$ \\
\hline 3077 & Mirandês & Mainland (Portugal) & $\mathrm{b} ; \mathrm{a} ; \mathrm{h} *$ \\
\hline 3153 & Alentejano & Mainland (Portugal) & $\mathrm{b} ; \mathrm{d} ; \mathrm{a}$ \\
\hline 2977 & Ardito & Mainland (Portugal) & $\mathrm{b} ; \mathrm{aj} ; \mathrm{a}$ \\
\hline 3844 & Galego rapado & Mainland (Portugal) & $\mathrm{b} ; \mathrm{g} ; \mathrm{a}$ \\
\hline 3094 & Santareno & Mainland (Portugal) & $\mathrm{b} ; \mathrm{f} ; \mathrm{g}$ \\
\hline 3081 & Temporão de Coruche & Mainland (Portugal) & $\mathrm{b} ; \mathrm{a} ; \mathrm{a}$ \\
\hline 3188 & Tremês ruivo & Mainland (Portugal) & $\mathrm{b} ; \mathrm{e} ; \mathrm{a}$ \\
\hline 3067 & Mocho ou rapado & Mainland (Portugal) & $\mathrm{b} ; \mathrm{f} ; \mathrm{d}$ \\
\hline 3105 & Grécia & Mainland (Portugal) & $\mathrm{b} ; \mathrm{g} ; \mathrm{c}$ \\
\hline ISOP00072 & T. Branco & Madeira (Portugal) & $\mathrm{b} ; \mathrm{a} / \mathrm{b} / \mathrm{f} ; \mathrm{a}$ \\
\hline ISOP00073 & T. Galhoto & Madeira (Portugal) & $\mathrm{b} / \mathrm{c} ; \mathrm{f} / \mathrm{h} ; \mathrm{a}$ \\
\hline ISOP00074 & T. Raposo & Madeira (Portugal) & $\mathrm{b} / \mathrm{c} ; \mathrm{a} / \mathrm{c} / \mathrm{f} ; \mathrm{a}$ \\
\hline ISOP00076 & T. Serra & Madeira (Portugal) & $\mathrm{b} ; \mathrm{d} / \mathrm{f} / \mathrm{h} / \mathrm{ae} ; \mathrm{a} / \mathrm{g}$ \\
\hline ISOP00077 & T. Vermelho & Madeira (Portugal) & $\mathrm{b} / \mathrm{c} ; \mathrm{b} / \mathrm{f} / \mathrm{ae} ; \mathrm{a}$ \\
\hline ISOP00079 & T. Cabeiro & Madeira (Portugal) & $\mathrm{b} / \mathrm{c} ; \mathrm{a} / \mathrm{b} / \mathrm{f} ; \mathrm{a}$ \\
\hline ISOP00080 & T. Preto & Madeira (Portugal) & $\mathrm{c} ; \mathrm{a} / \mathrm{b} / \mathrm{f} / \mathrm{g} / \mathrm{e} ; \mathrm{a} / \mathrm{b} / \mathrm{d}$ \\
\hline ISOP00083 & T. Barbela & Madeira (Portugal) & $\mathrm{b} / \mathrm{c} ; \mathrm{f} ; \mathrm{a}$ \\
\hline ISOP00084 & T. Pardo & Madeira (Portugal) & $\mathrm{b} / \mathrm{c} ; \mathrm{b} / \mathrm{f} / \mathrm{u} ; \mathrm{a}$ \\
\hline ISOP00085 & T. Leacock & Madeira (Portugal) & $\mathrm{b} / \mathrm{c} ; \mathrm{f} ; \mathrm{a}$ \\
\hline ISOP00087 & T. Leacock & Madeira (Portugal) & $\mathrm{b} / \mathrm{c} ; \mathrm{c} / \mathrm{f} ; \mathrm{a} / \mathrm{g}$ \\
\hline ISOP00089 & T. Raposo & Madeira (Portugal) & $\mathrm{b} / \mathrm{c} ; \mathrm{a} / \mathrm{e} / \mathrm{f} / \mathrm{g} ; \mathrm{a}$ \\
\hline ISOP00092 & T. Branco & Madeira (Portugal) & $\mathrm{b} / \mathrm{c} ; \mathrm{b} / \mathrm{f} ; \mathrm{a}$ \\
\hline ISOP00093 & T. Canalha & Madeira (Portugal) & $\mathrm{b} / \mathrm{c} ; \mathrm{a} / \mathrm{b} / \mathrm{f} ; \mathrm{a}$ \\
\hline ISOP00094 & T. Douradinho & Madeira (Portugal) & $\mathrm{b} ; \mathrm{a} / \mathrm{f} ; \mathrm{a} / \mathrm{g}$ \\
\hline ISOP00097 & T. Sem Pragana & Madeira (Portugal) & $\mathrm{b} / \mathrm{c} ; \mathrm{b} / \mathrm{d} / \mathrm{f} / \mathrm{g} / \mathrm{u} ; \mathrm{a} / \mathrm{b} / \mathrm{d} / \mathrm{g}$ \\
\hline ISOP00098 & T. Peladinho & Madeira (Portugal) & $\mathrm{b} / \mathrm{c} ; \mathrm{c} ; \mathrm{a} / \mathrm{b} / \mathrm{d} / \mathrm{g}$ \\
\hline ISOP00100 & T. Mouro & Madeira (Portugal) & $\mathrm{b} ; \mathrm{u} ; \mathrm{a}$ \\
\hline ISOP00101 & T. do Mato & Madeira (Portugal) & $\mathrm{b} ; \mathrm{f} ; \mathrm{a}$ \\
\hline ISOP00102 & T. Branco & Madeira (Portugal) & $\mathrm{b} ; \mathrm{b} / \mathrm{c} / \mathrm{f} ; \mathrm{a}$ \\
\hline ISOP00103 & T. Cana Roxa & Madeira (Portugal) & $\mathrm{b} ; \mathrm{a} / \mathrm{f} ; \mathrm{a}$ \\
\hline ISOP00105 & T. Branco & Madeira (Portugal) & $\mathrm{b} / \mathrm{c} ; \mathrm{f} ; \mathrm{a} / \mathrm{g}$ \\
\hline
\end{tabular}


Table 1 continued

\begin{tabular}{|c|c|c|c|}
\hline Accession number & Common name & Origin & Alleles \\
\hline ISOP00107 & T. Temporão & Madeira (Portugal) & $\mathrm{b} / \mathrm{c} ; \mathrm{b} ; \mathrm{a}$ \\
\hline ISOP00108 & T. Arroz & Madeira (Portugal) & $\mathrm{b} ; \mathrm{f} ; \mathrm{a}$ \\
\hline ISOP00110 & T. Rapadinho & Madeira (Portugal) & $\mathrm{b} / \mathrm{c} ; \mathrm{a} / \mathrm{f} ; \mathrm{a}$ \\
\hline ISOP00111 & T. Galhoto & Madeira (Portugal) & $\mathrm{b} / \mathrm{c} ; \mathrm{f} ; \mathrm{a} / \mathrm{g}$ \\
\hline ISOP00112 & T. Doiradinho & Madeira (Portugal) & $\mathrm{b} ; \mathrm{f} / \mathrm{g} ; \mathrm{a}$ \\
\hline ISOP00113 & T. do Porto Santo & Madeira (Portugal) & $\mathrm{b} ; \mathrm{b} / \mathrm{f} ; \mathrm{a}$ \\
\hline ISOP00114 & T. Rapado (Branco) & Madeira (Portugal) & $\mathrm{a} / \mathrm{b} / \mathrm{c} ; \mathrm{c} / \mathrm{e} / \mathrm{f} ; \mathrm{a} / \mathrm{b} / \mathrm{d} / \mathrm{g}$ \\
\hline ISOP00115 & T. Rapadinho & Madeira (Portugal) & $\mathrm{a} / \mathrm{b} / \mathrm{c} ; \mathrm{b} / \mathrm{c} / \mathrm{f} / \mathrm{ag} ; \mathrm{a} / \mathrm{b} / \mathrm{g}$ \\
\hline ISOP00116 & T. Rapado & Madeira (Portugal) & $\mathrm{b} / \mathrm{c} ; \mathrm{b} / \mathrm{g} / \mathrm{c} ; \mathrm{a} / \mathrm{g}$ \\
\hline ISOP00117 & T. Rapado & Madeira (Portugal) & $\mathrm{a} / \mathrm{b} / \mathrm{c} ; \mathrm{b} / \mathrm{c} ; \mathrm{a} / \mathrm{d}$ \\
\hline ISOP00118 & T. Rapadinho & Madeira (Portugal) & $\mathrm{b} ; \mathrm{a} / \mathrm{f} ; \mathrm{a}$ \\
\hline ISOP00119 & T. Raposinho & Madeira (Portugal) & $\mathrm{b} ; \mathrm{f} / \mathrm{g} ; \mathrm{a}$ \\
\hline ISOP00120 & T. Raposo & Madeira (Portugal) & $\mathrm{b} ; \mathrm{f} / \mathrm{g} ; \mathrm{a}$ \\
\hline ISOP00123 & T. Maçaroquinho & Madeira (Portugal) & $\mathrm{b} / \mathrm{c} ; \mathrm{b} / \mathrm{u} ; \mathrm{a}$ \\
\hline ISOP00238 & T. do Cedo & Madeira (Portugal) & $\mathrm{b} ; \mathrm{f} ; \mathrm{a}$ \\
\hline ISOP00239 & T. Rapado Branco & Madeira (Portugal) & $\mathrm{b} / \mathrm{c} ; \mathrm{f} / \mathrm{g} ; \mathrm{a}$ \\
\hline ISOP00241 & T. Novo & Madeira (Portugal) & $\mathrm{b} / \mathrm{c} ; \mathrm{d} / \mathrm{f} ; \mathrm{a} / \mathrm{g}$ \\
\hline ISOP00242 & T. de 3 meses & Madeira (Portugal) & $\mathrm{b} / \mathrm{c} ; \mathrm{b} ; \mathrm{a}$ \\
\hline ISOP00243 & T. Cana Roxa & Madeira (Portugal) & $\mathrm{b} / \mathrm{c} ; \mathrm{a} / \mathrm{f} / \mathrm{g} ; \mathrm{a}$ \\
\hline ISOP00246 & T. mais largo em cima & Madeira (Portugal) & $\mathrm{b} / \mathrm{c} ; \mathrm{a} / \mathrm{c} / \mathrm{d} / \mathrm{f} / \mathrm{g} / \mathrm{u} ; \mathrm{a} / \mathrm{b} / \mathrm{d}$ \\
\hline ISOP00247 & T. Mentana & Madeira (Portugal) & $\mathrm{b} / \mathrm{c} ; \mathrm{a} / \mathrm{b} ; \mathrm{a}$ \\
\hline ISOP00248 & T. Galhoto & Madeira (Portugal) & $\mathrm{b} / \mathrm{c} ; \mathrm{a} / \mathrm{b} / \mathrm{f} / \mathrm{g} ; \mathrm{a}$ \\
\hline ISOP00278 & T. Leacock & Madeira (Portugal) & $\mathrm{b} / \mathrm{c} ; \mathrm{b} ; \mathrm{a}$ \\
\hline ISOP00124 & T. Galhoto & Madeira (Portugal) & $\mathrm{b} ; \mathrm{a} ; \mathrm{a}$ \\
\hline ISOP00191 & T. Branco & Madeira (Portugal) & $\mathrm{b} / \mathrm{c} ; \mathrm{d} / \mathrm{f} ; \mathrm{a}$ \\
\hline ISOP00258 & T. Canoco & Madeira (Portugal) & $\mathrm{b} ; \mathrm{a} / \mathrm{f} ; \mathrm{a}$ \\
\hline ISOP00264 & T. Leacock sem P. & Madeira (Portugal) & $\mathrm{b} / \mathrm{c} ; \mathrm{b} / \mathrm{d} / \mathrm{g} ; \mathrm{a} / \mathrm{d}$ \\
\hline ISOP00288 & T. Potuguês & Madeira (Portugal) & $\mathrm{a} / \mathrm{b} ; \mathrm{c} ; \mathrm{a} / \mathrm{d}$ \\
\hline ISOP00007 & Trigo & Madeira (Portugal) & $\mathrm{b} / \mathrm{c} ; \mathrm{f} / \mathrm{g} ; \mathrm{a} / \mathrm{c} / \mathrm{d}$ \\
\hline ISOP00078 & T. Leacock & Madeira (Portugal) & $\mathrm{b} ; \mathrm{b} ; \mathrm{a}$ \\
\hline ISOP00025 & Trigo & Madeira (Portugal) & $\mathrm{c} ; \mathrm{f} ; \mathrm{b}$ \\
\hline ISOP00289 & T. Leacock com barbas & Madeira (Portugal) & $\mathrm{c} ; \mathrm{f} ; \mathrm{b}$ \\
\hline ISOP00319 & Trigo & Madeira (Portugal) & $\mathrm{b} / \mathrm{c} ; \mathrm{b} / \mathrm{g} ; \mathrm{a} / \mathrm{b} / \mathrm{d}$ \\
\hline ISOP01484 & T. rapadinho & Madeira (Portugal) & $\mathrm{b} ; \mathrm{b} / \mathrm{f} ; \mathrm{a}$ \\
\hline ISOP01357 & Trigo & Madeira (Portugal) & $\mathrm{b} / \mathrm{c} ; \mathrm{f} / \mathrm{g} ; \mathrm{a} / \mathrm{d}$ \\
\hline ISOP01485 & T. rapadinho de baixo Glúten & Madeira (Portugal) & $\mathrm{b} ; \mathrm{b} / \mathrm{f} ; \mathrm{a}$ \\
\hline ISOP01322 & T. Branco & Madeira (Portugal) & $\mathrm{b} / \mathrm{c} ; \mathrm{e} / \mathrm{f} ; \mathrm{a} / \mathrm{d}$ \\
\hline ISOP01269 & Trigo & Madeira (Portugal) & $\mathrm{b} ; \mathrm{a} ; \mathrm{a}$ \\
\hline ISOP01298 & Raposo & Madeira (Portugal) & $\mathrm{c} ; \mathrm{d} ; \mathrm{c}$ \\
\hline $1 *$ & Trigo & Canary Islands (Spain) & $\mathrm{a} / \mathrm{b} ; \mathrm{a} / \mathrm{d} / \mathrm{g} ; \mathrm{a}$ \\
\hline $2 *$ & Trigo Marroquín & Canary Islands (Spain) & $\mathrm{a} / \mathrm{b} ; \mathrm{b} / \mathrm{e} / \mathrm{u} ; \mathrm{d}$ \\
\hline $3 *$ & Trigo rápido & Canary Islands (Spain) & $\mathrm{a} / \mathrm{b} ; \mathrm{b} ; \mathrm{a} / \mathrm{d}$ \\
\hline $4^{*}$ & Trigo Blanco & Canary Islands (Spain) & $\mathrm{a} ; \mathrm{b} / \mathrm{u} / \mathrm{v} ; \mathrm{a} / \mathrm{d}$ \\
\hline $5 *$ & Trigo Peloño & Canary Islands (Spain) & $\mathrm{a} / \mathrm{b} ; \mathrm{b} / \mathrm{f} / \mathrm{g} ; \mathrm{a} / \mathrm{c} / \mathrm{d}$ \\
\hline
\end{tabular}


Table 1 continued

\begin{tabular}{|c|c|c|c|}
\hline Accession number & Common name & Origin & Alleles \\
\hline $6^{*}$ & Trigo morisco & Canary Islands (Spain) & $\mathrm{b} ; \mathrm{b} / \mathrm{d} / \mathrm{e} / \mathrm{u} ; \mathrm{g}$ \\
\hline 001 & Barbilla blanco & Canary Islands (Spain) & $\mathrm{b} ; \mathrm{d} ; \mathrm{a}$ \\
\hline 002 & Barbilla colorado & Canary Islands (Spain) & $\mathrm{b} ; \mathrm{d} ; \mathrm{a}$ \\
\hline 003 & Barbilla blanco & Canary Islands (Spain) & $\mathrm{b} ; \mathrm{d} ; \mathrm{a}$ \\
\hline 004 & Barbilla colorado & Canary Islands (Spain) & $\mathrm{b} ; \mathrm{d} / \mathrm{f} ; \mathrm{a}$ \\
\hline 005 & Barbilha Blanco & Canary Islands (Spain) & $\mathrm{b} ; \mathrm{d} ; \mathrm{a}$ \\
\hline 006 & Barbilla colorado & Canary Islands (Spain) & $\mathrm{b} ; \mathrm{b} / \mathrm{f} ; \mathrm{a}$ \\
\hline 007 & Barbilla & Canary Islands (Spain) & $\mathrm{b} ; \mathrm{d} / \mathrm{f} / \mathrm{h} ; \mathrm{a}$ \\
\hline 008 & Marrueco & Canary Islands (Spain) & $\mathrm{b} ; \mathrm{d} ; \mathrm{a}$ \\
\hline 009 & & Canary Islands (Spain) & $\mathrm{c} ; \mathrm{d} ; \mathrm{a}$ \\
\hline 010 & Marrueco & Canary Islands (Spain) & $\mathrm{c} ; \mathrm{a} / \mathrm{e} ; \mathrm{a}$ \\
\hline 011 & Colorado & Canary Islands (Spain) & $\mathrm{b} ; \mathrm{f} ; \mathrm{a}$ \\
\hline 012 & & Canary Islands (Spain) & $\mathrm{b} ; \mathrm{f} ; \mathrm{a}$ \\
\hline BGE18651 & Arisnegro de Tenerife & Canary Islands (Spain) & $\mathrm{b} ; \mathrm{a} / \mathrm{d} ; \mathrm{a}$ \\
\hline BGE24864 & Trigo & Canary Islands (Spain) & $\mathrm{b} ; \mathrm{d} ; \mathrm{a}$ \\
\hline BGE29104 & Trigo & Canary Islands (Spain) & $\mathrm{b} ; \mathrm{g} ; \mathrm{a}$ \\
\hline BGE29105 & Trigo & Canary Islands (Spain) & $\mathrm{b} ; \mathrm{f} ; \mathrm{g}$ \\
\hline BGE13760 & Isla de Fuerteventura & Canary Islands (Spain) & $\mathrm{b} ; \mathrm{f} ; \mathrm{a}$ \\
\hline BGE29103 & Trigo & Canary Islands (Spain) & $\mathrm{a} / \mathrm{b} ; \mathrm{a} / \mathrm{f} / \mathrm{h} ; \mathrm{a} / \mathrm{d}$ \\
\hline BGE18226 & T. colorado & Canary Islands (Spain) & $\mathrm{a} / \mathrm{b} ; \mathrm{a} / \mathrm{b} ; \mathrm{a} / \mathrm{d}$ \\
\hline BGE13754 & Morisco blanco & Canary Islands (Spain) & $\mathrm{b} ; \mathrm{f} ; \mathrm{a}$ \\
\hline BGE24863 & Trigo & Canary Islands (Spain) & $\mathrm{b} ; \mathrm{d} ; \mathrm{a}$ \\
\hline BGE13761 & Isla de Fuerteventura & Canary Islands (Spain) & $\mathrm{b} ; \mathrm{f} ; \mathrm{g}$ \\
\hline BGE13160 & Morisco rojo & Canary Islands (Spain) & $\mathrm{b} ; \mathrm{d} / \mathrm{f} / \mathrm{g} ; \mathrm{a}$ \\
\hline BGE31122 & Trigo de la tierra & Canary Islands (Spain) & $\mathrm{b} / \mathrm{c} ; \mathrm{b} ; \mathrm{a}$ \\
\hline BGE31123 & Trigo pelon & Canary Islands (Spain) & $\mathrm{b} ; \mathrm{v} ; \mathrm{a}$ \\
\hline BGE20366 & T. alto & Canary Islands (Spain) & $\mathrm{b} / \mathrm{c} ; \mathrm{d} / \mathrm{e} ; \mathrm{a} / \mathrm{g}$ \\
\hline СBT00724 & T. di alto & Canary Islands (Spain) & b; aj/f/g; a \\
\hline СВТ00685 & T. de alto & Canary Islands (Spain) & $\mathrm{b} / \mathrm{c} ; \mathrm{d} / \mathrm{f} ; \mathrm{a}$ \\
\hline СВТ00684 & T. Jallado & Canary Islands (Spain) & $\mathrm{b} / \mathrm{c} ; \mathrm{b} / \mathrm{d} / \mathrm{f} ; \mathrm{a}$ \\
\hline СВТ00686 & & Canary Islands (Spain) & $\mathrm{b} / \mathrm{c} ; \mathrm{d} / \mathrm{f} ; \mathrm{a}$ \\
\hline СВТ00527 & T. barbilla marrueco & Canary Islands (Spain) & $\mathrm{b} / \mathrm{c} ; \mathrm{d} / \mathrm{f} / \mathrm{g} ; \mathrm{a}$ \\
\hline СВТ00524 & Barbilla blanco & Canary Islands (Spain) & $\mathrm{b} ; \mathrm{d} / \mathrm{f} ; \mathrm{a}$ \\
\hline СBT00522 & Barbilla blanco & Canary Islands (Spain) & $\mathrm{b} ; \mathrm{b} / \mathrm{d} / \mathrm{f} ; \mathrm{a} / \mathrm{d}$ \\
\hline СВТ00598 & & Canary Islands (Spain) & $\mathrm{b} / \mathrm{c} ; \mathrm{a} / \mathrm{d} / \mathrm{f} ; \mathrm{a}$ \\
\hline СВT00622 & Barbilla & Canary Islands (Spain) & $\mathrm{b} ; \mathrm{d} / \mathrm{f} ; \mathrm{a}$ \\
\hline СВТ00608 & Barbilla & Canary Islands (Spain) & $\mathrm{b} ; \mathrm{d} ; \mathrm{a}$ \\
\hline СВT00398 & Morisco & Canary Islands (Spain) & $\mathrm{b} ; \mathrm{b} / \mathrm{d} / \mathrm{f} ; \mathrm{a}$ \\
\hline СВТ00391 & & Canary Islands (Spain) & $\mathrm{b} ; \mathrm{f} ; \mathrm{a} / \mathrm{d}$ \\
\hline СBT00306 & T. colorado & Canary Islands (Spain) & $\mathrm{b} ; \mathrm{f} ; \mathrm{a} / \mathrm{d}$ \\
\hline СВТ00528 & Barbilla marrueco & Canary Islands (Spain) & $\mathrm{b} / \mathrm{c} ; \mathrm{a} / \mathrm{d} / \mathrm{e} / \mathrm{g} ; \mathrm{a}$ \\
\hline СВТ00379 & T. morisco & Canary Islands (Spain) & $\mathrm{b} ; \mathrm{b} / \mathrm{e} ; \mathrm{a}$ \\
\hline СВТ00384 & & Canary Islands (Spain) & $\mathrm{b} ; \mathrm{f} ; \mathrm{a}$ \\
\hline СBT00307 & Plaganudo & Canary Islands (Spain) & $\mathrm{a} / \mathrm{b} / \mathrm{c} ; \mathrm{a} / \mathrm{b} / \mathrm{d} ; \mathrm{a}$ \\
\hline
\end{tabular}


Table 1 continued

\begin{tabular}{|c|c|c|c|}
\hline Accession number & Common name & Origin & Alleles \\
\hline СВТ00383 & T. del país & Canary Islands (Spain) & $\mathrm{b} ; \mathrm{d} / \mathrm{h} / \mathrm{an} ; \mathrm{a}$ \\
\hline СВT00521 & Barbilla colorado & Canary Islands (Spain) & b; b/f/aj; a \\
\hline CBT00520 & Barbilla blanco & Canary Islands (Spain) & $\mathrm{a} / \mathrm{b} ; \mathrm{d} / \mathrm{f} ; \mathrm{a}$ \\
\hline СВT00243 & Barbilla & Canary Islands (Spain) & $\mathrm{b} ; \mathrm{d} / \mathrm{f} ; \mathrm{a}$ \\
\hline СВТ00242 & Arisnegro de Tenerife & Canary Islands (Spain) & $\mathrm{b} / \mathrm{c} ; \mathrm{a} / \mathrm{b} / \mathrm{e} / \mathrm{f} / \mathrm{i} ; \mathrm{a}$ \\
\hline СBT00241 & Arisnegro & Canary Islands (Spain) & $\mathrm{b} / \mathrm{c} ; \mathrm{d} / \mathrm{f} / \mathrm{ag} ; \mathrm{a}$ \\
\hline СВT00240 & Marroquí & Canary Islands (Spain) & $\mathrm{b} / \mathrm{c} ; \mathrm{b} / \mathrm{d} / \mathrm{f} / \mathrm{i} ; \mathrm{a} / \mathrm{d}$ \\
\hline СBT00377 & Marroquí & Canary Islands (Spain) & $\mathrm{b} / \mathrm{c} ; \mathrm{d} / \mathrm{f} ; \mathrm{a}$ \\
\hline СВТ00293 & Morisco & Canary Islands (Spain) & $\mathrm{b} ; \mathrm{d} / \mathrm{g} ; \mathrm{a}$ \\
\hline CBT00308 & Marrueco & Canary Islands (Spain) & $\mathrm{b} ; \mathrm{a} / \mathrm{b} / \mathrm{d} / \mathrm{g} ; \mathrm{a}$ \\
\hline СВТ00239 & Colorado de Tacoronte & Canary Islands (Spain) & $\mathrm{b} ; \mathrm{a} / \mathrm{d} / \mathrm{f} ; \mathrm{a}$ \\
\hline СBT00244 & Arisnegro & Canary Islands (Spain) & $\mathrm{b} / \mathrm{c} ; \mathrm{a} / \mathrm{d} / \mathrm{g} ; \mathrm{a}$ \\
\hline СBT00523 & Barbilla colorado & Canary Islands (Spain) & $\mathrm{b} ; \mathrm{d} / \mathrm{f} ; \mathrm{a}$ \\
\hline СВТ00526 & T. barbilla marrueco & Canary Islands (Spain) & b/c; f/aj; a \\
\hline СBT00623 & T. barbilla & Canary Islands (Spain) & $\mathrm{b} ; \mathrm{d} / \mathrm{f} ; \mathrm{a}$ \\
\hline СВТ00309 & Marsello & Canary Islands (Spain) & $\mathrm{b} ; \mathrm{b} / \mathrm{d} / \mathrm{f} / \mathrm{g} / \mathrm{an} ; \mathrm{a}$ \\
\hline 146 & Trigo peloño & Canary Islands (Spain) & $\mathrm{a} / \mathrm{b} / \mathrm{c} ; \mathrm{a} / \mathrm{b} / \mathrm{f} ; \mathrm{a} / \mathrm{d} / \mathrm{g}$ \\
\hline 148 & Trigo rápido & Canary Islands (Spain) & $\mathrm{a} / \mathrm{b} ; \mathrm{a} / \mathrm{b} ; \mathrm{d}$ \\
\hline 149 & Trigo blanco & Canary Islands (Spain) & $\mathrm{a} / \mathrm{b} / \mathrm{c} ; \mathrm{a} / \mathrm{b} ; \mathrm{a} / \mathrm{d}$ \\
\hline 150 & Trigo peloño & Canary Islands (Spain) & $\mathrm{a} / \mathrm{c} ; \mathrm{b} / \mathrm{e} / \mathrm{f} ; \mathrm{a} / \mathrm{d}$ \\
\hline 151 & Trigo marroquin & Canary Islands (Spain) & $\mathrm{b} ; \mathrm{b} / \mathrm{I} ; \mathrm{d}$ \\
\hline 152 & Trigo Blanco & Canary Islands (Spain) & $\mathrm{a} / \mathrm{b} ; \mathrm{b} ; \mathrm{c}$ \\
\hline 215 & - & Canary Islands (Spain) & $\mathrm{b} ; \mathrm{e} ; \mathrm{a}$ \\
\hline 217 & Trigo pelón & Canary Islands (Spain) & $a ; b ; d$ \\
\hline 222 & Trigo elón & Canary Islands (Spain) & $c ; f ; a$ \\
\hline
\end{tabular}

(ne), Shannon's information index (S), average heterozygosity, genetic diversity (He), genetic differentiation (Fst) and. Gene flow (Nm).

The genetic identify (I) values were calculated among the populations. The Nei's genetic distances (D) were used to generate a clustered dendrogram based on unweighted pair-group method using the MEGA version 4 program (Tamura et al. 2007).

\section{Results}

The frequencies of 25 alleles identified at the 3 loci encoding HMWGs are shown in Table 2. Three alleles were identified at $G l u-A 1$, sixteen at the $G l u-B 1$ and six at the Glu-Dl locus. At the Glu-Al locus, three alleles were detected in the wheat accessions originated from the Canary and Madeira Islands and two from the continental Portugal (Table 2). Glu-Alb was the most common allele with a frequency of $84.4 \%$ among the accessions from continental Portugal, $79.35 \%$ among the Canary accessions and $65.82 \%$ among the Madeira accessions. The frequency of $\mathrm{Glu}$ Ala was $15.6 \%$ in the Portuguese accessions, $11.4 \%$ in the Canary accessions and only $1.38 \%$ in the accessions from Madeira. On the other hand, the allele Glu$A 1 c$ had the highest frequency of $32.8 \%$ in the Madeira wheats followed by $9.24 \%$ in the Canary wheats, while it was not detected in wheat accessions from the continental Portugal.

In wheat accessions originated from the Canary Island, the allele $f$ of $G l u$-B1locus was the most frequent allele $(31.43 \%)$ followed by Glu-Bld $(29.43 \%)$. In the Madeira wheat the Glu-Blf and Glu-Blb loci were the more frequent alleles, representing 47.09 and $16.51 \%$ of landraces, respectively. In the continental accessions the Glu-Bld locus shown the highest frequency $(24.77 \%)$ followed by Glu-Bla 
Table 2 The frequencies (\%) of high molecular weight glutenin subunits (HMWGS) in bread wheat (Triticum aestivum L.)

\begin{tabular}{|c|c|c|c|c|c|}
\hline Locus & Allele & Subunits type & Canary & Madeira & $\begin{array}{l}\text { Portugal } \\
\text { (mainland) }\end{array}$ \\
\hline & $\mathrm{a}$ & 1 & 11.40 & 1.38 & 15.60 \\
\hline & $\mathrm{b}$ & $2 *$ & 79.35 & 65.82 & 84.40 \\
\hline \multirow[t]{7}{*}{ Glu-Al } & $\mathrm{c}$ & Null & 9.24 & 32.80 & \\
\hline & $\mathrm{a}$ & 7 & 5.24 & 11.01 & 17.43 \\
\hline & $\mathrm{b}$ & $7+8$ & 15.72 & 16.51 & 8.17 \\
\hline & $\mathrm{c}$ & $7+9$ & & 8.47 & \\
\hline & d & $6+8$ & 29.43 & 2.65 & 24.77 \\
\hline & $\mathrm{e}$ & 20 & 3.85 & 2.75 & 7.34 \\
\hline & $\mathrm{f}$ & $13+16$ & 31.43 & 47.09 & 15.60 \\
\hline \multirow[t]{13}{*}{ Glu-B1 } & $\mathrm{g}$ & $13+19$ & 6.16 & 5.93 & 14.68 \\
\hline & $\mathrm{h}$ & $14+15$ & 1.08 & 0.32 & \\
\hline & $\mathrm{i}$ & $17+18$ & 1.08 & & \\
\hline & an & 6 & 0.31 & & 5.5 \\
\hline & aj & 8 & 1.23 & & 4.59 \\
\hline & ae & $18^{*}$ & & 0.63 & \\
\hline & & $6+9$ & & 0.53 & \\
\hline & ag & $7 *$ & 0.31 & 0.63 & 0.92 \\
\hline & $\mathrm{u}$ & $7 *+8$ & 2.62 & 2.75 & \\
\hline & af & $7 *+9$ & 1.54 & & \\
\hline & $\mathrm{a}$ & $2+12$ & 80.28 & 80.53 & 54.12 \\
\hline & $\mathrm{b}$ & $3+12$ & & 6.03 & \\
\hline & $\mathrm{c}$ & $4+12$ & 1.23 & 0.95 & 4.59 \\
\hline \multirow[t]{3}{*}{ Glu-D1 } & d & $5+10$ & 12.33 & 6.24 & 9.17 \\
\hline & $\mathrm{g}$ & $2+10$ & 6.16 & 6.24 & 23.85 \\
\hline & $\mathrm{h}$ & $2+12 *$ & & & 8.26 \\
\hline
\end{tabular}

$(17.43 \%)$. The subunit type $18 *, 7+9$ and $6+9$ were detected only in the Madeira wheat and the alleles Glu-B1i, Glu-Blan, Glu-Blaj and Glu-Blaf (Fig. 1) were absent in the Madeira accessions. The Glu-Bli and Glu-Blaf (Fig. 1) alleles were present only in the Canary wheat, while the continental accessions shown only nine of the seventeen alleles found at the Glu-B1 locus.

At the Glu-D1 locus, six alleles were detected with Glu-Dla being the preponderant allele in the Canary $(80.28 \%)$, Madeira $(80.53 \%)$ and in the continental $(54.12 \%)$ accessions. The Glu-Dl $h$ allele was detected only in the Old Portuguese wheat $(8.26 \%)$.

The allele Glu-Dld associated with good bread making quality (Payne et al. 1987) was detected among the Canary, Madeira and mainland Portuguese accessions, showing the highest frequency in the wheat originated from the Canary Islands $(12.33 \%)$. Subunit $3+12(G l u-D 1 b)$ was present only in Madeira accessions (6.03\%) (Fig. 1) whereas $4+12$ (GluD1c) (Fig. 1) appeared in all the three regions, with a low frequency of $1.23,0.95$ and $4.59 \%$ the Canary, Madeira and continental Portugal accessions, respectively. The low frequency of these two subunits can be an advantage, because there are associated with poor bread making quality (Payne et al. 1987). A total of 69 allele combinations were observed in our data, with the most common being Glu-Alb/Glu-Blf/Glu-Dla.

The genetic data for the Glu-l loci are summarized in the Table 3. The effective number of alleles (ne), the expected heterozygosity $(\mathrm{He})$ and the Shannon information index shown the highest values at the GluAl loci in the wheat originated from Madeira. The highest value of genic diversity (Fst) detected among in the wheat accessions from the continental Portugal, while similar values of gene flow in all loci were found in the Madeira and in Portuguese wheat's described by Vasconcellos (1933). 
Fig. 1 SDS-PAGE patterns of HMW-GS from some accessions, representative of the most uncommon allelic variants detected at the Glu-1 loci. Lanes as follows: 1 (Florida 301); 2 (Gabo); 3 (Marquis); 4 (Florence);

5 (CBT00308);

6 (ISOP00097); 7 (3172);

8 (ISOP00089);

9 (Federation); 10 (006);

11(CBT00240); 12 (2*);

$13(1 *) ; 14$ (Champlein);

15 (152); 16 (ISOP00098);

17 (ISOP00093); 18 (3077);

19 (ISOP00248);

20 (CBT00526);

21 (ISOP00105);

22 (Marquis);

23 (ISOP00288);

24 (ISOP00072);

25 (ISOP00087);

26 (CBT00309)
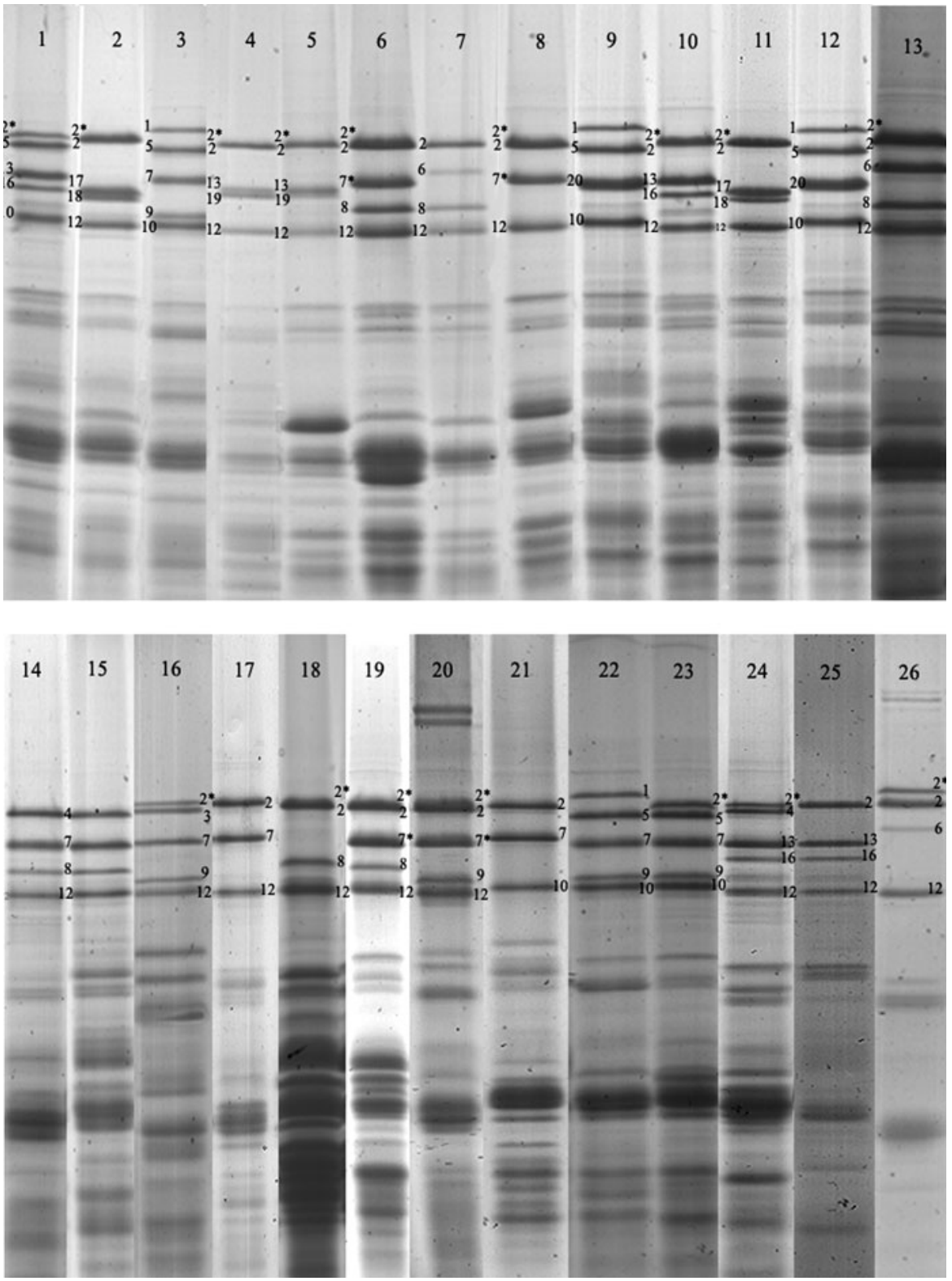

At the Glu-BI locus the Portuguese wheat's (the Vasconcellos collection) shown the highest values for effective number of alleles (ne), the Shannon information index (S), expected heterozygosity $(\mathrm{He})$ and genic diversity (Fst). In general the wheat's from the Madeira and Canary Islands exhibited a similar variation of genetic parameters.

At the Glu-Dl locus the Canary and Madeira wheat accessions had similar values, while the Portuguese ones shown higher levels of genetic variations except average heterozygosity.
The mean genic diversity (Fst) for al loci were 0.54 (Madeira), 0.66 (Canary) and 0.92 (Old Portuguese) wheat's. These results indicate a high diversity among the populations from the three different geographical regions.

A UPGMA dendrogram was constructed based on Nei's (1973) genetic distance for all populations (Fig. 2). All populations were grouped into two main clusters (I and II). While the majority of accessions were included in the cluster II, the accession 152 and 00010 originating from Canary (La Palma) Islands 


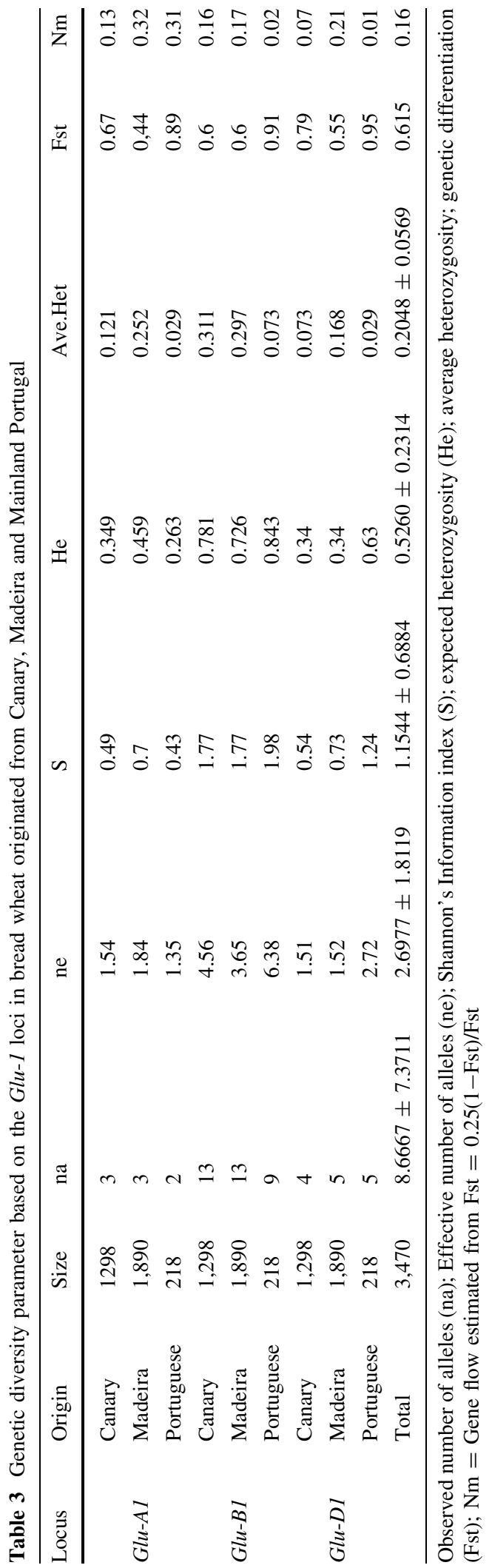

were contained within the cluster I. The cluster II was divided into 33 subclusters, of which eight encompassed accessions from Madeira (subcluster \# 5, 8, 11, 14, 21, 23, 24 and 27), five contained accessions from Canary (subcluster \# 13, 16, 19, 26 and 32), nine had accessions from Madeira and Canary (subcluster \# 2, $3,6,10,12,22,25,28$ and 31), six contained accessions from Madeira, Canary and Portugal (subcluster \# 9, 15, 17, 18, 29 and 30) and five included wheat accessions from Canary and Portugal (subcluster \# 1, 4, 7, 20 and 33).

\section{Discussion}

The analysis of variation in storage protein can provide important information for the assessment of genetic variability of plant germplasms (Igrejas et al. 1999).

The accessions analyzed in this study included landraces from Madeira (Portugal), the Canary Islands (Spain) and the continental Portugal. Twenty six alleles were detected at all loci. The 69 haplotypes were found at the HMW glutenin loci owing to the combination of the allelic variants at Glu-A1, Glu-B1 and Glu-D1. The Glu-Alb/Glu-Blf/Glu-Dla haplotype was the most frequent in all three geographical regions. The average genic diversity (Fst) in each locus displayed high values, which points to the existence of genic variability among the Madeira, Canary and Portuguese (the Vasconcellos collection) wheat populations and to a low level of immigration. The latter suggests that the populations are well structured and are of a different geographical origin.

The cluster analysis confirmed the historic information about the introduction and the spread of wheat across the studied regions. Initially, wheat was introduced by settlers from Portugal to the Island of Madeira and the majority of the land was dedicated to this crop. Excess of crop yield left after satisfying the needs of local population was sold to the mainland of Portugal, Maghreb, Azores and the Canary Islands. The Madeiran wheat grain was also used to support the Portuguese exploration of the cost of northern of Africa and in the Gulf of Guinea (de Albuquerque and Vieira 1987; Vieira 1987, 1988). The cluster 9, 15, 17, 18,29 and 30 seem to match with this historic data. From the seventies of the fifteenth century, a great part of the land was converted to sugar cane and grape 


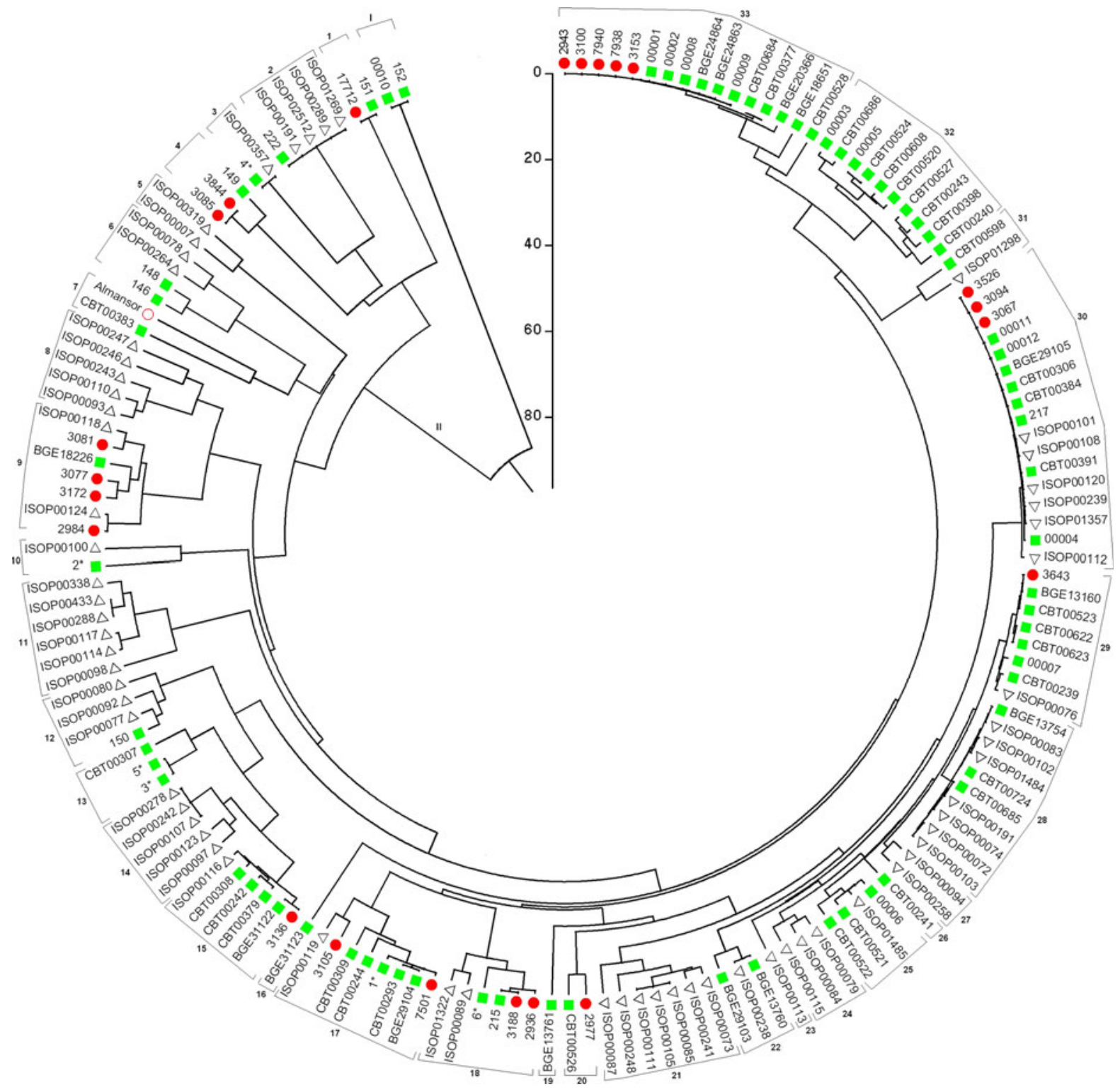

Fig. 2 Dendrogram based on unweighted pair-group method. The accessions marked with filled circle originated from mainland Portugal, with open triangle from Madeira, and with filled square from Canary Islands

production and wheat cultivation was transferred to less adequate land, which resulted in substantial reduction of wheat yields. To compensate for decline of wheat production on Madeira new fertile areas in the region were identified and put into cultivation including the Azores Islands and the Canary Islands (Vieira 1984). Subsequently, in the XV, XVI and XVII centuries an intensive trade was development among the archipelagos of Madeira, Canary and Azores. Madeira imported wheat from Azores and the
Canary Island and exported for both archipelagos sugar and wine (Vieira 1983, 1984; Freitas de Meneses 1995). The subcluster 2, 3, 6, 10 and 12 included landraces from Madeira and La Palma (Canary Islands), which reflects the wheat trade activities between the two islands. La Palma had played an important role in the commerce of wheat to Madeira and it was a popular immigration destination of residents of Madeira who introduced the sugar production technology to this island (Vieira 1984). 
Fig. 3 The routes of the wheat exchange among Iberian Peninsula and the Atlantic Islands (Azores, Madeira and Canary) and north of Africa. In the fifteenth century the wheat was introduced in Madeira from Mainland (1), in the seventies was export from this Island to mainland Portugal (2), Azores (3) and Canary Islands (4). In the end of fifteenth and sixteenth and seventeenth centuries Madeira imported wheat from Azores (5) and Canary Islands (8); Canary Islands (6) and North Africa (7) imported wheat from Azores Islands in the sixteenth and seventeenth centuries

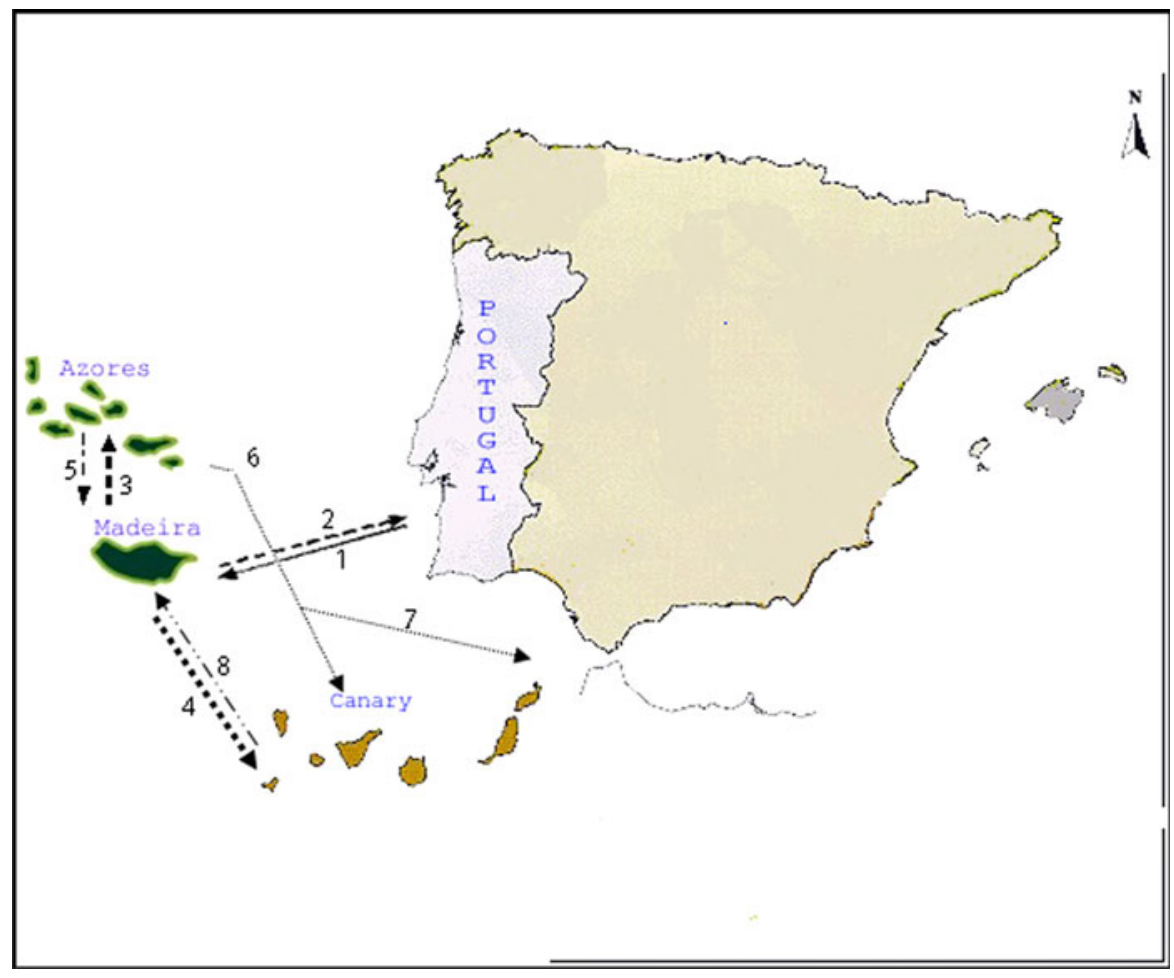

Tenerife, the other island of the Archipelago of Canary, has also played an important role in the commercial exchanges with Madeira. This historical fact could be supported by the analysis of clusters 15 , 22, 25 and 28. The Branch I includes only sample from La Palma, which may suggest another origin of these samples. The three archipelagos (Madeira, Azores and Canaries) for several centuries have played a key role in the navigations and connections among Europe, America and Asia. All exchanges of wheat grain that happened during this period led to the accumulation of a great agricultural diversity on these islands, which is now stored in germplasm banks in Madeira and Canary islands. To prevent the genetic erosion produced by genetic drift in accessions stored in germplasm banks as result of their management (Hammer 2003), it is important to promote the utilisation of these local landraces by the farmers.

We propose that geographical distribution of the high molecular weight glutenin alleles confirms the existence of wheat exchanges from the Iberian Peninsula to Madeira and from Madeira to the Canary Islands and vice versa (Fig. 3). Also, the high genetic diversity among the population of each island supports the hypothesis of different wheat introductions to both archipelagos. Data from the Azores Islands were not included because wheat as a crop is presently extinct in this region.

Acknowledgments This research was supported by the European Community through the project AGRICOMAC and GERMOBANCO INTERREG III-B. We thank to Bank of Germplasm INIA (CRF, Alcalá de Henares, Spain), to Maria Teresa Carvalho e Vasconcelos from Instituto Superior de Agronomia (Lisbon) and the National Small Grain Collection (Aberdeen, USA) for supplying the wheat accessions.

\section{References}

Branlard G, Dardevet M, Amiour N, Igrejas G (2003) Allelic diversity of HMW and LMW glutenin subunits and omega gliadins in French bread wheat (Triticum aestivum L.). Genet Resour Crop Evol 50:669-679

Caballero L, Martin LM, Alvarez JB (2001) Allelic variation of the HMW glutenin subunits in Spanish accessions of spelta wheat (Triticum aestivum ssp. spelta L. em. Thell.). Theor Appl Genet 103:124-128

Chaparzadeh N, Sofalian O, Javanmard A, Hejazi MS, Zarandi L (2008) Study of glutenin subunits in some wheat landraces from northwest of Iran by SDS-PAGE technique. Int J Agri Biol 10:101-104

Cooke RJ (ed) (1992) Electrophoresis handbook: variety identification. In: Handbook of variety testing. International Seed Testing Association, Zurich, pp 1-50 
de Albuquerque L, Vieira A (1987) O Arquipélago da Madeira no Século XV. Secretaria Regional do Turismo e Cultura, Funchal

dos Santos TMM, Ganança F, Slaski J, Pinheiro de Carvalho MAA (2009) Morphological characterization of wheat genetic resources from the Island of Madeira, Portugal. Genet Resour Crop Evol 56:363-375

Feldman M (2000) Origin of cultivated wheat. In: Bonjean AP, Angus WJ (eds) The world wheat book: a history of wheat breeding. Intercept Ltd., London, pp 3-56

Feldman M, Lupton FGH, Miller TE (1995) Wheats. In: Smartt J, Simmonds NW (eds) Evolution of crop plants. Longman group Ltd., London, pp 184-192

Feng D, Xia G, Zhao S, Chen F (2004) Two quality-associated HMW glutenin subunits in a somatic hybrid line between Triticum aestivum and Agropyron elongatum. Theor Appl Genet 110:136-144

Freitas de Meneses A (1995) Os Açores nas encruzilhadas de Setecentos (1740-1770), II. Economia. Universidade dos Açores, Ponta Delgada, pp. 31-77 e 166-191

Hammer K (2003) A paradigm shift in the discipline of plant genetic resources. Genet Resour Crop Evol 50:3-10

Hua C, Takata K, Yang-Fen Z, Ikeda TM, Yanaka M, Nagamine T, Fujimaki H (2005) Novel high molecular weight glutenin subunits at the Glu-Dl locus in wheat landraces from the Xinjiang District of China and relationship with winter habit. Breed Sci 55:459-463

Igrejas G, Guedes-Pinto H, Carnide V, Branlard G (1999) The high and low molecular weight glutenin subunits and $<$ omega $>$-gliadin composition of bread and durum wheats commonly grown in Portugal. Plant Breed 118:297-302

Lagudah ES, Flood RG, Halloran GM (1987) Variation in high molecular weight glutenin subunits in landraces of hexaploid wheat from Afghanistan. Euphytica 36:3-9

Lawrence GJ (1986) The high-molecular-weight glutenin subunit composition of Australian wheat cultivars. Aust $\mathbf{J}$ Agric Res 37:125-133

Lawrence GL, Shepherd KW (1981) Inheritance of glutenin protein subunits of wheat. Theor Appl Genet 60:333-337

Li Y, Huang C, Sui X, Fan Q, Li G, Chu X (2009) Genetic variation of wheat glutenin subunits between landraces and varieties and their contributions to wheat quality improvement in china. Euphytica 169:159-168

Morgunov AI, Rogers WJ, Sayers EJ, Metakovsky EV (1990) The high-molecular-weight glutenin subunit composition of Soviet wheat varieties. Euphytica 51:41-52

Nei M (1973) Analysis of gene diversity in subdivided populations. Proc Natl Acad Sci USA 70:3321-3323

Nevo E, Payne PI (1987) Wheat storage protein diversity of HWM glutenin subunits in wild emmer from Isreal. 1. Geographical patterns and ecogeographical predicabality. Theor Appl Genet 74:827-836

Payne PI (1987) Genetic of wheat storage proteins and the effect of allelic variation on breadmaking quality. Ann Rev Plant Physiol 38:147-153

Payne PI, Lawrence GJ (1983) Catalogue of alleles for the complex loci, Glu-A1, Glu-B1 and Glu-D1 which coded for high-molecular-weight subunits of glutenin in hexaploid wheat. Cereal Res Commun 11:29-35

Payne PI, Nightingale A, Krattinger AF, Holt LM (1987) The relationship between HMW glutenin subunit composition and the bread making quality of British-grown wheat varieties. J Sci Food Agric 40:51-65

Porceddu E, Turchetta T, Masci S, D’Ovidio R, Lafiandra D, Kasarda DD, Impiglia A, Nachit MM (1998) Variation in endosperm protein composition and technological quality properties in durum wheat. Euphytica 100(1-3):197-205

Shan XY, Clayshulte SR, Haley SD, Byrne PF (2007) Variation for glutenin and waxy alleles in the US hard winter wheat germplasm. J Cereal Sci 45:199-208

Shewry PR, Napier JA, Tatham AS (1995) Seed storage proteins: structures and biosynthesis. Plant Cell 7:945-956

Tamura K, Dudley J, Nei M, Kumar S (2007) MEGA4: molecular evolutionary genetics analysis (MEGA) software version 4.0. Mol Biol Evol 24:1596-1599

Terasawa Y, Kawahara T, Sasakuma T, Sasanuma T (2009) Evaluation of the genetic diversity of an Afghan wheat collection based on morphological variation, HMW glutenin subunit polymorphisms, and AFLP. Breed Sci 59:361-371

Tohver M (2007) High molecular weight (HMW) glutenin subunit composition of some Nordic and Middle European wheats. Genet Resour Crop Evol 54:67-81

Uthayakumaran S, Listiohadi Y, Baratta M, Batey IL, Wrigley CW (2006) Rapid identification and quantification of highmolecular-weight glutenin subunits. J Cereal Sci 44:34-39

Vallega V (1988) Comparative analysis of high-molecularweight glutenin subunit composition in various Triticum species. Plant Breed 100:241-246

Vasconcellos JC (1933) Trigos Portugueses. Boletim de Agricultura, series I, no. 1-2, pp 150

Vieira A (1983) O comércio de cereais dos Açores para a Madeira no século XVII, vol XLI. Separata do Boletim do Instituto Histórico da Ilha Terceira, Ilha Terceira, pp 651-677

Vieira A (1984) O Comércio de Cereais das Canárias para a Madeira nos Séculos XVI e XVII. In: VI Colóquio de História Canário Americana, Las Palmas, Casa de Colon, Funchal, pp 327-351

Vieira A (1987) O Comércio inter-insular nos séculos XV e XVI: Madeira, Açores e Canárias. Secretaria Regional do Turismo e Cultura. Centro de Estudos de História do Atlântico, Funchal

Vieira A (1988) A Madeira na Rota dos Descobrimentos e a Expansão Atlântica, vol XXXIV. Instituto de Investigação Científica Tropical, Separata da Revista da Universidade de Coimbra, Lisboa, pp 571-580

Wan Y, Yan Z, Liu K, Zheng Y, D'Ovidio R, Shewry PR, Halford NG, Wang D (2005) Comparative analysis of the D genome-encoded high-molecular weight subunits of glutenin. Theor Appl Genet 111:1183-1190

Yeh FC, Boyle TJB (1997) Population genetic analysis of codominant and dominant markers and quantitative traits. Bel J Bot 129:157 